\title{
A Non-Stationary IMT-Advanced MIMO Channel Model for High-Mobility Wireless Communication Systems
}

\author{
Ammar Ghazal, Student Member, IEEE, Yi Yuan, Cheng-Xiang Wang, Senior Member, IEEE, \\ Yan Zhang, Member, IEEE, Qi Yao, Hongrui Zhou, and Weiming Duan
}

\begin{abstract}
With the recent developments of high-mobility wireless communication systems, e.g., high-speed train (HST) and vehicle-to-vehicle (V2V) communication systems, the ability of conventional stationary channel models to mimic the underlying channel characteristics has widely been challenged. Measurements have demonstrated that the current standardized channel models, like IMT-Advanced (IMT-A) and WINNER II channel models, offer stationary intervals that are noticeably longer than those in measured HST channels. In this paper, we propose a non-stationary channel model with time-varying parameters including the number of clusters, the powers and the delays of the clusters, the angles of departure (AoDs), and the angles of arrival (AoAs). Based on the proposed non-stationary IMT-A channel model, important statistical properties, i.e., the local spatial crosscorrelation function (CCF) and local temporal autocorrelation function (ACF) are derived and analyzed. Simulation results demonstrate that the statistical properties vary with time due to the non-stationarity of the proposed channel model. An excellent agreement is achieved between the stationary interval of the developed non-stationary IMT-A channel model and that of relevant HST measurement data, demonstrating the utility of the proposed channel model.
\end{abstract}

Index Terms-IMT-Advanced, MIMO, non-stationary, timevarying parameters, statistical properties.

\section{INTRODUCTION}

Multiple-input multiple-output (MIMO) has attracted a lot of attention recently as a promising technique that can enhance the system performance and link reliability. Wireless channel modeling plays an important role on designing and evaluating MIMO wireless communication systems. Demonstrating the

The authors acknowledge the support from Huawei Technologies, International S\&T Cooperation Program of China (Grant No. 2014DFA11640), EPSRC TOUCAN project (Grant No. EP/L020009/1), EU H2020 5G Wireless project (Grant No. 641985), EU FP7 QUICK project (Grant No. PIRSES-GA2013-612652), 863 project in 5G, Ministry of Science and Technology (Grant No. 2014AA01A707), and Natural Science Foundation of China (Grant No. 61210002).

A. Ghazal is with the School of Engineering and Sustainable Development, De Montfort University, Leicester, UK (e-mail: ammar-ghazal@ outlook.com).

Y. Yuan is with Ocado Technology, UK (e-mail: yyuan1116@gmail.com).

C.-X. Wang (corresponding author), and Q. Yao are with the Institute of Sensors, Signals and System, School of Engineering and Physical Sciences, Heriot-Watt University, Edinburgh, EH14 4AS, UK. C.-X. Wang is also with Shandong Provincial Key Lab of Wireless Communication Technologies, School of Information Science and Engineering, Shandong University, Jinan, 250100, China (e-mail: cheng-xiang.wang@hw.ac.uk, qiyao@ hotmail.com).

Y. Zhang is with the School of Information and Electronics, Beijing Institute of Technology, Beijing 100081, China (e-mail: zhangy@bit.edu.cn).

H. Zhou and W. Duan are with the Shanghai Research Institute, Huawei Technologies Co., Ltd., Shanghai, 201206, China (e-mail: \{zhouhongrui, weiming.duan\}@huawei.com). feasibility of wireless systems is not possible without accurate channel models that are able to mimic key characteristics of the wireless channels.

Many standardized MIMO channel models are available in the literature, e.g., IEEE 802.11 TGn channel model [1], long-term evolution-advanced (LTE-A) channel model [2], 3GPP/3GPP2 spatial channel model (SCM) [3], SCMExtension (SCME) [4], WINNER I [5], WINNER II [6], WINNER+ [7], and IMT-Advanced (IMT-A) [8] channel models. However, most of the aforementioned standardized channel models have neglected the non-stationarity of the fading channels assuming that modeled channels satisfy the wide sense stationary (WSS) assumption. The WINNER II, WINNER+, and IMT-A channel models have introduced the concept of time-evolution to explicitly simulate the non-stationarity of the fading channels but they fell short when compared with real measured channels, as we will introduce later in Section V.C.

High-mobility wireless communication systems face the challenge of providing reliable communication services over rapidly changing radio channels in hostile environments such as tunnels [9] and subways [10]. Practical measurement campaigns [11]-[14] have proved that the WSS assumption is only valid for short intervals considering the time variation of real wireless channels. Measurement data reported in [15] has demonstrated that the stationary interval, defined as the maximum time duration over which the channel satisfies the WSS condition, of standard channel models, like IMT-A and WINNER II, is considerably longer than that of a real highspeed train (HST) channel. In [16], measurement of vehicle-tovehicle $(\mathrm{V} 2 \mathrm{~V})$ channels has proved that the stationary interval of channels between vehicles driving in opposite directions can be very short. Therefore, research of non-stationary channel modeling has gradually become a hot research topic especially for high-mobility scenarios that are expected to be typical scenarios for the fifth generation $(5 \mathrm{G})$ wireless communication systems [17].

Different modeling approaches have been introduced in the literature to model non-stationary channels. Non-stationary V2V and HST channel models were surveyed in [18] and [19], respectively, whilst non-stationary massive MIMO channel models were reviewed in [20], [21]. Non-stationary geometrybased deterministic models (GBDMs) were proposed in [22], [23] to model V2V channels and in [24] to model HST channels. GBDMs incorporate detailed simulations of the actual physical wave propagation process for a given en- 
vironment. Therefore, they are physically meaningful and potentially accurate. Despite their accuracy, GBDMs only consider specific propagation scenarios and require detailed descriptions of the propagation environments and extensive computational resources to be implemented. Non-geometrical stochastic models (NGSMs) characterize physical parameters of propagation channels in a completely stochastic manner by providing their underlying probability distribution functions without assuming an underlying geometry. In [25] and [26], the authors proposed NGSMs for non-stationary V2V and HST channels, respectively. NGSMs are convenient to characterize the death/birth properties of the moving clusters (MCs) using Markov chains. However, the statistical properties of the nonstationary NGSMs are difficult to derive. In geometry-based stochastic models (GBSMs), the impulse responses of HST channels are characterized by the law of wave propagation applied to specific transmitter, receiver, and scatterers' geometries which are predefined in a stochastic fashion according to certain probability distributions. Time-variant GBSMs with time-varying parameters were proposed in [27]-[29] for V2V channels and in [30], [31] for HST channels where some interesting local statistical properties were derived by taking time-varying parameters into account. However, these existing time-variant GBSMs are only modeled for some specific scenarios, such as specified moving directions for MCs and mobile stations (MSs) [27], specified roadside conditions [28], [29], and specific environments [30], [31].

In conventional cellular wireless network, the base station (BS) is fixed while a MS is moving towards a certain direction with a constant speed. In non-stationary IMT-A channel models, we assume that the movements of MCs and MSs result in the time-variant properties. Following the trajectory of the MCs and a MS, we can obtain small-scale parameters (SSPs), such as the number of clusters, the mean power and delay of each cluster (power delay profile (PDP)), angles of departure (AoDs), and angles of arrival (AoAs). The lack of implementation for non-stationarity IMT-A MIMO channel models and the aforementioned research gaps inspire us to propose a non-stationary channel model with the aforementioned timevarying parameters.

We summarize the major contributions and novelties of this paper as follows:

(1) A non-stationary IMT-A channel model with timevarying SSPs, i.e., the number of clusters, delays, the power of each cluster, AoDs, and AoAs, is proposed.

(2) Based on the proposed non-stationary IMT-A channel model, important statistical properties, i.e., the local spatial cross-correlation function (CCF) and local temporal autocorrelation function (ACF) are derived and analyzed.

(3) The stationary interval of the proposed non-stationary IMT-A channel model is investigated and compared with the one of the standard IMT-A channel model and with a measurement data.

The rest of this paper is structured as follows. In Section II, the original IMT-A MIMO channel model is briefly introduced. Section III presents the proposed novel non-stationary IMT-A channel models with various time-varying SSPs. The local statistical properties of the non-stationary IMT-A channel model with time-varying parameters are derived in Section IV and then simulated and analyzed in Section V. Finally, the conclusions are drawn in Section VI.

\section{OVERVIEW OF THE ORIGINAL IMT-A MIMO CHANNEL MODEL}

For the evaluation of IMT-A systems, the ITU has defined a channel model [8] that consists of pathloss models, scenario-specific parameter sets for the indoor hotspot (InH), urban micro-cell (UMi), urban macro-cell (UMa), rural macrocell (RMa), and suburban macro-cell (SMa) scenarios, and a so-called generic model. The generic model specifies the mathematical model and the algorithms used for channel modeling that applied to all scenarios. It is based on the WINNER II channel model [6]. This generic model employs a GBSM approach to represent the multi-path propagation channel between a BS and MSs.

In IMT-A channel model, the channel is assumed to satisfy the wide sense stationary uncorrelated scattering (WSSUS) assumption which means, in time domain, that the channel fading statistics remain invariant over a short period of time and that the scatterers with different path delays are uncorrelated. Please note that the WSSUS assumption is only valid to describe the short-term variations of the radio channel. Based on the WSSUS assumption and the tapped delay line (TDL) concept, the original IMT-A MIMO channel model has the complex channel impulse response (CIR) between the BS antenna element $s(s=1, \ldots, S)$ and MS antenna element $u$ $(u=1, \ldots, U)$ as [8]:

$$
h_{u, s}(t, \tau)=\sum_{n=1}^{N} h_{u, s, n}(t) \delta\left(\tau-\tau_{n}\right)
$$

where

$$
\begin{aligned}
& h_{u, s, n}(t)=\sqrt{\frac{P_{n}}{M}} \sum_{m=1}^{M} e^{j d_{s} k \sin \left(\phi_{n, m}\right)} e^{j d_{u} k \sin \left(\varphi_{n, m}\right)} \\
& \times e^{j 2 \pi v_{n, m} t} e^{j \Phi_{n, m}} .
\end{aligned}
$$

Here, $h_{u, s, n}(t)(n=1, \ldots, N)$ denotes a narrowband process where all the $M$ sub-paths within each of the $N$ clusters are irresolvable rays and have the same delay $\tau_{n}, P_{n}$ is the power of the $n$th cluster associated with the delay $\tau_{n}, d_{s}$ and $d_{u}$ are the antenna element spacings at the BS and MS, respectively, $k=\frac{2 \pi}{\lambda}$ is the wave number where $\lambda$ is the carrier wavelength, $\phi_{n, m}$ and $\varphi_{n, m}$ denote the AoD and AoA related to the $m$ th $(m=1, \ldots, M)$ ray within the $n$th $(n=$ $1, \ldots, N)$ cluster, respectively, and the random phases $\Phi_{n, m}$ are uniformly distributed within $[-\pi, \pi)$. The Doppler frequency component is represented by $v_{n, m}=\frac{\left\|\vec{v}_{M S}\right\| \cos \left(\varphi_{n, m}-\theta_{M S}\right)}{\lambda}$, where $\left\|\vec{v}_{M S}\right\|$ and $\theta_{M S}$ denote the magnitude of the MS velocity and MS direction of travel, respectively. All of the aforementioned parameters are time-invariant because of the WSSUS assumption in the original IMT-A channel model.

\section{A NOVEL NON-STATIONARY IMT-A CHANNEL MODEL WITH TIME-VARYING PARAMETERS}

In practice, because of the observable movement of MCs and/or MSs, the WSSUS assumption may be violated and 
the channel model parameters will change over the time. In this case, the complex CIR of the new non-stationary IMT-A channel model with time-varying parameters can be expressed as

$$
h_{u, s}(t, \tau)=\sum_{n=1}^{N(t)} h_{u, s, n}(t) \delta\left(\tau-\tau_{n}(t)\right)
$$

where

$$
\begin{array}{r}
h_{u, s, n}(t)=\sqrt{\frac{P_{n}(t)}{M}} \sum_{m=1}^{M} e^{j d_{s} k \sin \left(\phi_{n, m}(t)\right)} e^{j d_{u} k \sin \left(\varphi_{n, m}(t)\right)} \\
\times e^{j k\left\|\vec{v}_{M S}\right\| \cos \left(\varphi_{n, m}(t)-\theta_{M S}\right) t} e^{j \Phi_{n, m}} .
\end{array}
$$

Due to the movement of the MSs and/or MCs, the channel model parameters $N(t), \tau_{n}(t), P_{n}(t), \phi_{n, m}(t)$, and $\varphi_{n, m}(t)$ will change over the time and therefore they need to be represented using proper time-varying functions. Fig. 1 illustrates the proposed non-stationary IMT-A channel model, which is derived from the original IMT-A channel model [8]. The parameters in Fig. 1 are defined in Table I. The propagation between the first and the last interacted cluster is not defined. Thus, this approach can also model multiple interactions with the scattering media. Note that $\phi_{n, m}\left(t_{0}\right), \varphi_{n, m}\left(t_{0}\right), \Delta \phi_{n, m}$, and $\Delta \varphi_{n, m}$ denote the initial AoD, initial AoA, AoD offset, and AoA offset, respectively. The subscripts $n, m$ indicate one of the $N$ paths and one of the $M$ rays/sub-paths in the wideband channel, respectively. The AoD relates to the first bounce/cluster, i.e., Cluster $A$, interacted from the BS side and AoA is from the last bounce/cluster, i.e., Cluster $Z$. Furthermore, $\phi_{\mathrm{LoS}}$ and $\varphi_{\mathrm{LoS}}$ are the line-of-sight $(\mathrm{LoS})$ components for BS and MS, respectively. In Fig. 1, we assume that the $\mathrm{BS}$ is fixed while the first cluster, Cluster $A$, is in motion with the direction $\theta_{A}$ and speed $v_{A}$. As we consider multi-bounced scattering occurring for each path, Cluster $Z$ refers to the last bounce $\mathrm{MC}$, with the moving direction $\theta_{Z}$ and speed $v_{Z}$. For the MS, $\theta_{M S}$ is the moving direction with the speed $v_{M S}$. The movement of MC $A, \mathrm{MC} Z$, and MS are represented in Fig. 1 using the vectors $\vec{v}_{A}, \vec{v}_{Z}$, and $\vec{v}_{M S}$, respectively. Furthermore, $D_{\mathrm{BS}}\left(t_{0}\right)$ and $D_{\mathrm{MS}}\left(t_{0}\right)$, which represent the initial distance between the $\mathrm{BS}$ and $\mathrm{MC} A$, and the initial distance between the MS and MC $Z$, respectively, are assumed to be known. The initial distance of the LoS components between BS and MS is donated as $D_{\mathrm{LoS}}\left(t_{0}\right)$. To derive the time-varying angular parameters, we set four assistant angles, i.e., $\alpha_{n, m}(t), \beta, \gamma_{n, m}(t)$, and $\delta$ in Fig. 1. It should be noted that in Fig. 1, only the LOS path and the $n$th scattered propagation path are shown. As an example, Cluster $A$ and Cluster $Z$ are corresponding to the first bounce and the last bounce clusters of the $n$th scattered path, respectively. The speed $v_{A}$ of the MC $A$ and/or the speed $v_{A}$ of the MC $Z$ can be set as zero if MC $A$ and/or MC $Z$ is static. This will not affect the generation procedure of channel coefficients in the proposed non-stationary IMT-A MIMO channel model.

Based on the aforementioned geometric description, the channel realizations of the proposed non-stationary IMT-A channel model are obtained by the procedure illustrated in Fig. 2. Initial values of the model parameters, i.e., $N\left(t_{0}\right)$, $\tau_{n}\left(t_{0}\right), P_{n}\left(t_{0}\right), \phi_{n, m}\left(t_{0}\right)$, and $\varphi_{n, m}\left(t_{0}\right)$, will be calculated in a

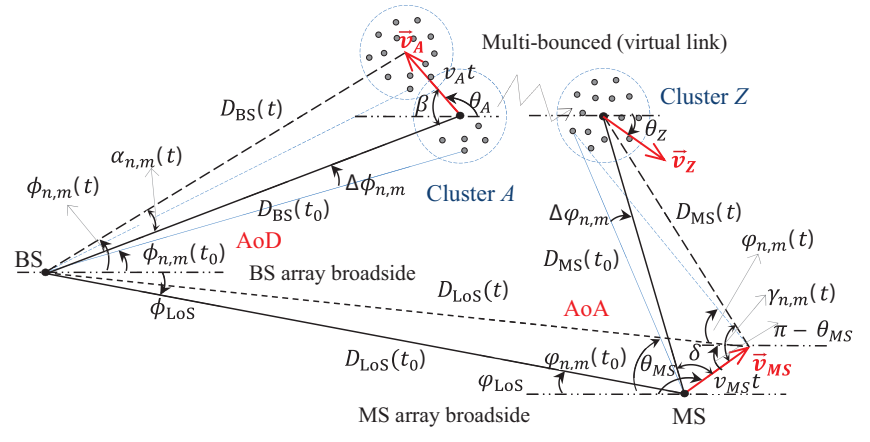

Fig. 1. BS and MS angular parameters in the IMT-A channel model.

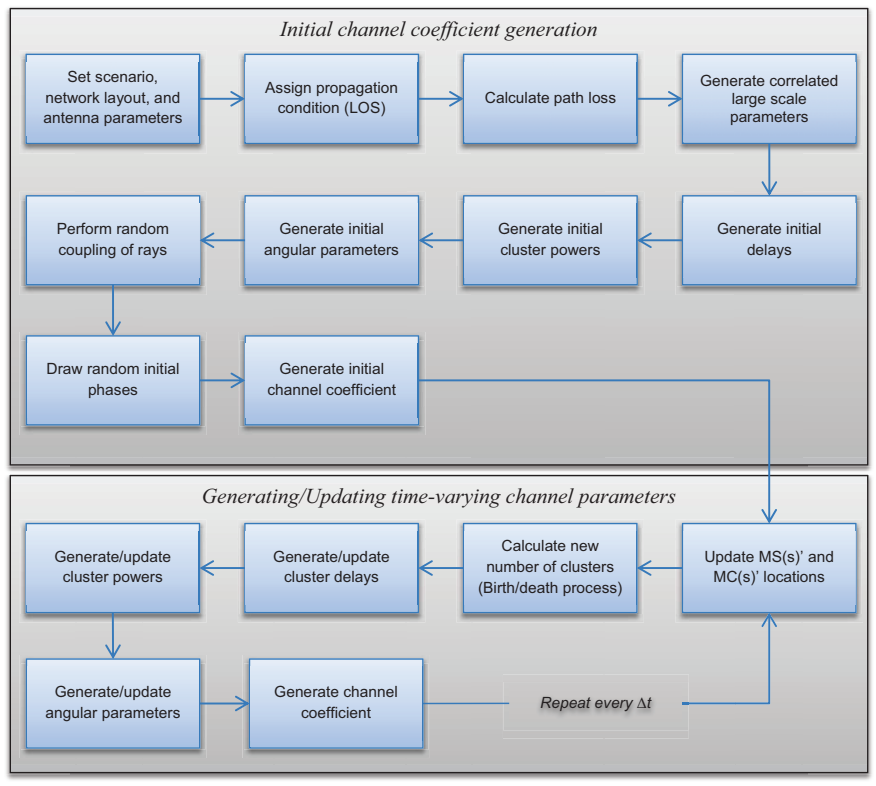

Fig. 2. The non-stationary IMT-A channel coefficients generation procedure.

similar procedure to the one presented in the IMT-A channel model (the top section of Fig. 2). Here, we will focus on the non-stationary properties of the proposed IMT-A channel model (the bottom section of Fig. 2).

\section{A. Update MCs and MS locations}

Based on the movement direction of $\mathrm{MC} A, \mathrm{MC} Z$, and $\mathrm{MS}$, the distances between $\mathrm{BS}$, MCs, and MS will change with time $t$. By considering the concept of relative velocity MC $Z$ and MS, we can assume that the MS is only in motion with a velocity relative to MC $Z$. As the velocity is a vector, which is equivalent to a specification of its speed and direction of motion, we define the velocity of MC $Z$ as $\vec{v}_{Z}=v_{Z} \cdot e^{j \theta_{Z}}$ and that of MS as $\vec{v}_{M S}=v_{M S} \cdot e^{j \theta_{M S}}$.

We assume that the MC $Z$ is fixed as a reference point, then the velocity of MS relative to the MC $Z$ can be expressed as

$$
\begin{aligned}
& \vec{v}_{M S / Z}=\vec{v}_{M S}-\vec{v}_{Z} \\
& =v_{M S} \cdot e^{j \theta_{M S}}-v_{Z} \cdot e^{j \theta_{Z}} \\
& =\left(v_{M S} \cdot \cos \theta_{M S}-v_{Z} \cdot \cos \theta_{Z}\right)+j\left(v_{M S} \cdot \sin \theta_{M S}-v_{Z} \cdot \sin \theta_{Z}\right) .
\end{aligned}
$$


TABLE I

DEFINITIONS OF CHANNEL PARAMETERS IN FIG. 1.

\begin{tabular}{|c|l|}
\hline Parameters & Definition \\
\hline$D_{\mathrm{BS}}(t)$ & distance between the BS and MC $A$ \\
\hline$D_{\mathrm{MS}}(t)$ & distance between the MS and MC $Z$ \\
\hline$D_{\mathrm{LoS}}(t)$ & distance of the LoS components between BS and MS \\
\hline$\phi_{n, m}(t), \varphi_{n, m}(t)$ & the AoD and AoA related to the $m$ th ray within the $n$th cluster, respectively \\
\hline$\phi_{L o S}, \varphi_{L o S}$ & the LoS AoD and LoS AoA, respectively \\
\hline$\Delta \phi_{n, m}, \Delta \varphi_{n, m}$ & the AoD offset and AoA offset, respectively \\
\hline$\vec{v}_{A}, \vec{v}_{Z}, \vec{v}_{M S}$ & the velocity vectors of MC $A$, MC $Z$, and MS, respectively \\
\hline$v_{A}, \theta_{A}$ & the MC $A$ velocity and MS direction of travel, respectively \\
\hline$v_{Z}, \theta_{Z}$ & the MC $Z$ velocity and MS direction of travel, respectively \\
\hline$v_{M S}, \theta_{M S}$ & the MS velocity and MS direction of travel, respectively \\
\hline
\end{tabular}

$$
\begin{gathered}
D_{\mathrm{BS}}(t)=\sqrt{D_{\mathrm{BS}}^{2}\left(t_{0}\right)+\left(v_{A} t\right)^{2}-2 \cdot D_{\mathrm{BS}}\left(t_{0}\right) \cdot v_{A} t \cdot \cos \left(\pi+\phi_{n, m}\left(t_{0}\right)-\theta_{A}\right)} \\
D_{\mathrm{MS}}(t)=\sqrt{D_{\mathrm{MS}}^{2}\left(t_{0}\right)+(v t)^{2}-2 \cdot a\left(t_{0}\right) \cdot v t \cdot \cos \left(\varphi_{n, m}\left(t_{0}\right)-\theta_{v}\right)} \\
D_{\mathrm{LoS}}(t)=\sqrt{D_{\mathrm{LoS}}^{2}\left(t_{0}\right)+\left(v_{M S} t\right)^{2}-2 \cdot D_{\mathrm{LoS}}\left(t_{0}\right) \cdot v_{M S} t \cdot \cos \left(\theta_{M S}-\varphi_{\mathrm{LoS}}\right)} .
\end{gathered}
$$

Therefore, $v_{M S / Z}$ and $\theta_{M S / Z}$ can be calculated by taking the magnitude and argument of $\vec{v}_{M S / Z}$, i.e., $v_{M S / Z}=$ $\left\|\vec{v}_{M S / Z}\right\|$ and $\theta_{M S / Z}=\arg \left(\vec{v}_{M S / Z}\right)$, respectively.

Assuming that the initial distances $D_{\mathrm{BS}}\left(t_{0}\right), D_{\mathrm{MS}}\left(t_{0}\right)$, and $D_{\mathrm{LoS}}\left(t_{0}\right)$ are obtained from a measurement campaign, the time-varying distances at time $t$ can then be expressed as (6), (7), and (8).

\section{$B$. The number of clusters, $N(t)$}

With the movement of the MS, the propagation environment between the MS and BS will change. Also, some moving scatterers, e.g., cars and walking people, will move within the environment. Thus, some paths can disappear and some new paths can appear. A genetic appearance (birth) and disappearance (death) process for modeling the clusters, or multi-path components (MPCs), was proposed in [32]. In a time-variant (i.e., non-stationary) scenario, clusters may only exist over a certain time period. With ongoing time, new clusters appear, i.e., born, and remain for a certain time span, i.e., survive, and then finally disappear, i.e., died. A suitable description for such a generation-recombination behavior is given by discrete Markov processes. The time variance of a wireless channel is mainly caused by movements of MCs and MS. Based on the process of clusters' generation and recombination, the time dependent channel fluctuations caused by the movement of the MCs $\left(\delta_{M C, k}\right)$ as well as the movement of MS $\left(\delta_{M S, k}\right)$ in the time span between $t_{k-1}$ and $t_{k}$, can be expressed as

$$
\delta_{P, k}=\delta_{M C, k}+\delta_{M S, k}
$$

with

$$
\delta_{M C, k}=\int_{t_{k-1}}^{t_{k}} P_{c}\left(\left\|\vec{v}_{Z}(t)\right\|+\left\|\vec{v}_{A}(t)\right\|\right) \mathrm{d} t
$$

and

$$
\delta_{M S, k}=\int_{t_{k-1}}^{t_{k}}\left(\left\|\vec{v}_{M S}(t)\right\|\right) \mathrm{d} t
$$

where $P_{c}$ is a percentage of moving clusters. Because of the short time steps, a uniform motion in each time interval $\mid t_{k}-$ $t_{k-1} \mid$ is assumed [32], [33]. This simplifies (10) and (11) to

$$
\delta_{M C, k}=\left(t_{k}-t_{k-1}\right) P_{c}\left(v_{Z}+v_{A}\right)
$$

$$
\delta_{M S, k}=\left\|\vec{v}_{M S}\left(t_{k}\right)-\vec{v}_{M S}\left(t_{k-1}\right)\right\|=\left(t_{k}-t_{k-1}\right) v_{M S} .
$$

Therefore, $\delta_{P, k}$ gives a measure for the correlation of CIRŁ at different time snapshots. A Markov birth-death description results in a time-varying number $N(t)$ of clusters for CIR realizations. At any time instant $t_{k}$, one can distinguish between newly generated clusters and survived clusters that were already existing in the previous CIR at time instant $t_{k-1}$. The Markov process is described by a generation rate of clusters $\left(\lambda_{G}\right)$ and a recombination rate of new paths $\left(\lambda_{R}\right)$. The expectation of the total number, also defined as the initial number, of clusters in a CIR realization is given by

$$
\mathbf{E}\{N(t)\}=N\left(t_{0}\right)=\frac{\lambda_{G}}{\lambda_{R}} .
$$

Observing a time series of CIRs, each cluster remains from one CIR at $t_{k-1}$ to a following one at $t_{k}$ with the probability

$$
P_{\text {remain }}\left(\delta_{P, k}\right)=e^{-\lambda_{R} \cdot \delta_{P, k}} \text {. }
$$

Hence, a number of new clusters is generated by the Markov process with expectation

$$
\mathbf{E}\left\{N_{\text {new }, k}\right\}=\frac{\lambda_{G}}{\lambda_{R}}\left(1-e^{-\lambda_{R} \cdot \delta_{P, k}}\right) .
$$

The correlation between two evolving clusters is quantified by the scenario movement $\delta_{P, k}$. Mathematically, higher values of $\delta_{P, k}$ result in a reduced correlation between the properties 


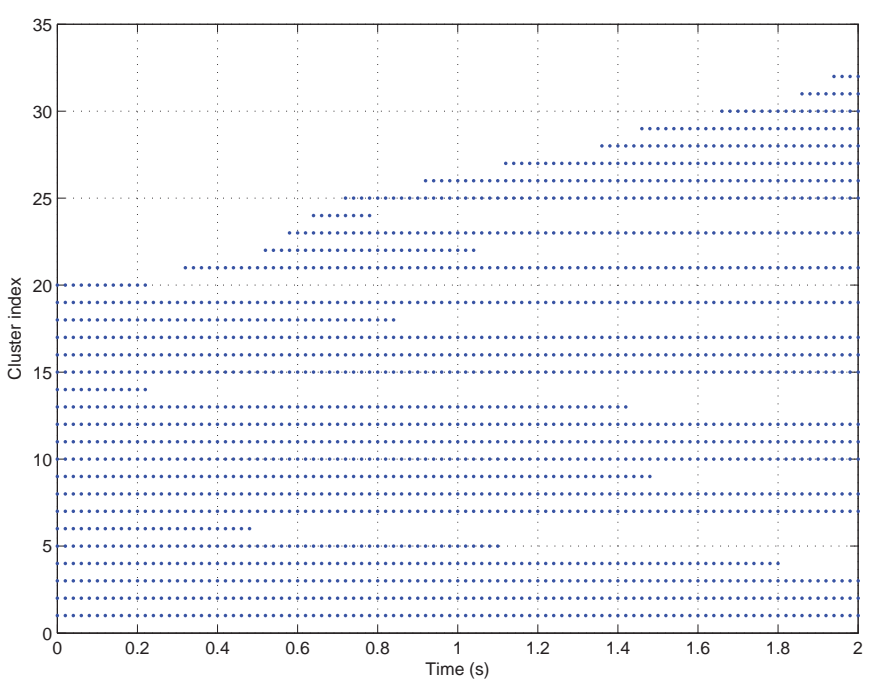

Fig. 3. Time-varying clusters with death/birth process. $\left(N\left(t_{0}\right)=20, \lambda_{G}=\right.$ $0.8 / \mathrm{m}, \lambda_{R}=0.04 / \mathrm{m}, v_{M S}=60 \mathrm{~m} / \mathrm{s}, v_{A}=15 \mathrm{~m} / \mathrm{s}, v_{Z}=5 \mathrm{~m} / \mathrm{s}$, and $\left.P_{c}=0.3\right)$

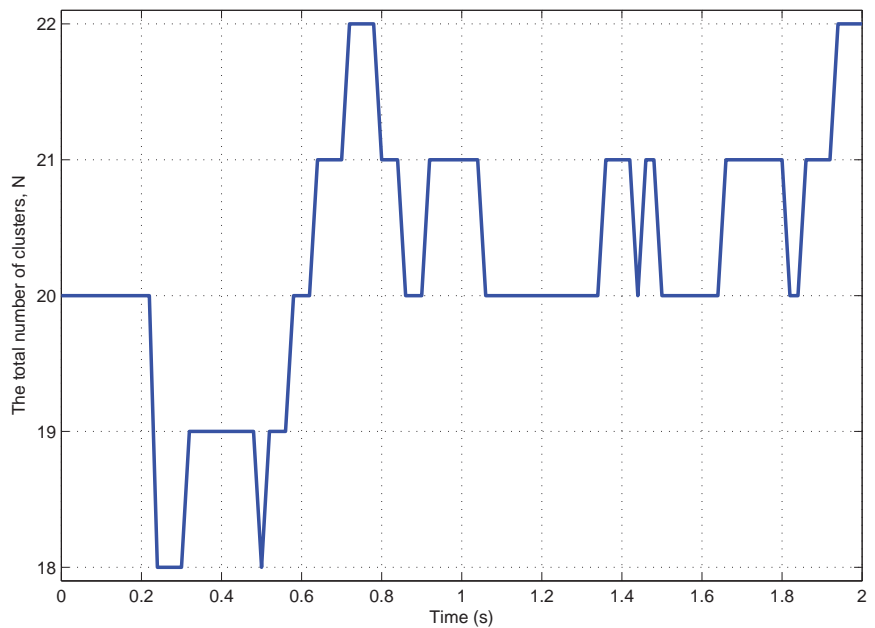

Fig. 4. The total number of clusters $N(t)$ vs. time. $\left(N\left(t_{0}\right)=20, \lambda_{G}=0.8 /\right.$ $\mathrm{m}, \lambda_{R}=0.04 / \mathrm{m}, v_{M S}=60 \mathrm{~m} / \mathrm{s}, v_{A}=15 \mathrm{~m} / \mathrm{s}, v_{Z}=5 \mathrm{~m} / \mathrm{s}$, and $P_{c}=$ $0.3)$

of an ancestor cluster at $t_{k-1}$ and its successor at $t_{k}$. Fig. 3 illustrates the time-variation of the number of clusters with birth-death process at the initial number of clusters $N\left(t_{0}\right)=$ 20 taken from IMT-A channel models at NLoS UMa scenario assuming $\lambda_{G}=0.8 / \mathrm{m}, \lambda_{R}=0.04 / \mathrm{m}, v_{M S}=60 \mathrm{~m} / \mathrm{s}, v_{A}=$ $15 \mathrm{~m} / \mathrm{s}, v_{Z}=5 \mathrm{~m} / \mathrm{s}$, and $P_{c}=0.3$. In Fig. 3, starting from the initial status $\left(N\left(t_{0}\right)=20\right)$, clusters are appear/disappear with the time going. In addition, Fig. 4 shows the time-varying number of clusters $N(t)$.

For the newly generated clusters, the delays, powers, and angular parameters will be generated similar to the initial parameters based on the procedure illustrated in Fig. 2 and explained in [8]. For the surviving clusters, the delays, powers, and angular parameters will be updated as follows.

\section{Time-varying delays, $\tau_{n}(t)$}

With the movements of MCs (including MC $A$ and MC $Z$ ) and MS, the propagation distance of the $n$th cluster will be time-variant and can be determined by using some trigonometric functions on the basis of the initial distance of the $n$th cluster as (6)-(8). The calculation of the time-varying delay $\tau_{n}(t)$ can be expressed as follows:

$$
\begin{aligned}
& \tau_{n}(t)=\frac{\Delta D}{c_{0}}+\tilde{\tau_{n}}(t)=\frac{\Delta D_{\mathrm{BS}}+\Delta D_{\mathrm{MS}}}{c_{0}}+\tilde{\tau_{n}}(t) \\
= & \frac{\left(D_{\mathrm{BS}}(t)-D_{\mathrm{BS}}\left(t_{0}\right)\right)+\left(D_{\mathrm{MS}}(t)-D_{\mathrm{MS}}\left(t_{0}\right)\right)}{c_{0}}+\tilde{\tau_{n}}(t)
\end{aligned}
$$

where $c_{0}$ is the speed of light, $\Delta D=\Delta D_{\mathrm{BS}}+\Delta D_{\mathrm{MS}}$ is the alteration of the propagation distance resulted from the movement of the MS and MCs. $\Delta D_{\mathrm{BS}}=D_{\mathrm{BS}}(t)-D_{\mathrm{BS}}\left(t_{0}\right)$ is the distance difference between $\mathrm{BS}$ and $\mathrm{MC} A, \Delta D_{M S}=$ $D_{\mathrm{MS}}(t)-D_{\mathrm{MS}}\left(t_{0}\right)$ is the distance difference between MC $Z$ and MS, and $\tilde{\tau_{n}}(t)$ is the delay of the virtual link between MC $A$ and MC $Z$. The delay of the virtual link, i.e., $\tilde{\tau_{n}}$, can be calculated at time instant $t=t_{k}$ using a first-order filtering algorithm as [34]

$$
\tilde{\tau_{n}}\left(t_{k}\right)=e^{\left(\left(t_{k-1}-t_{k}\right) / \varsigma\right)} \tilde{\tau_{n}}\left(t_{k-1}\right)+\left(1-e^{\left(\left(t_{k-1}-t_{k}\right) / \varsigma\right)}\right) X
$$

where $X \sim U\left(D_{\mathrm{LoS} / c_{0}}, \tau_{\max }\right), \varsigma$ is a parameter that depends on the coherence of a virtual link and scenarios, and $\tau_{\max }$ is the maximum delay and can be obtained from [8], e.g., $\tau_{\max }$ $=1885 \mathrm{~ns}$ for UMa Non-LoS (NLoS) scenario.

It is worth to be noted that $\tilde{\tau_{n}}\left(t_{0}\right)$ can be calculated as

$$
\tilde{\tau_{n}}\left(t_{0}\right)=\tau_{n}\left(t_{0}\right)-\frac{D_{\mathrm{BS}}\left(t_{0}\right)+D_{\mathrm{MS}}\left(t_{0}\right)}{c_{0}}
$$

where the initial delay $\tau_{n}\left(t_{0}\right)$ are randomly drawn from the exponential delay distribution, as explained in the original IMT-A channel models, for a given scenario [8]. Substituting (6), (7), (8) into (17), we therefore can obtain the timevarying delays for $n$th cluster at different instant time $t$, i.e., $\tau_{n}(t)$. Delays are then normalized by subtracting the minimum delay and sort the normalized delays to descending order. The normalized delays are to be used in channel coefficient generation (3) but not in cluster power generation.

\section{Time-varying power of clusters, $P_{n}(t)$}

Cluster powers are calculated assuming a single slope exponential power delay profile. The random averaged power for the $n$th cluster at time $t_{0}$, denoted as $P_{n}\left(t_{0}\right)$, can be generated from the parameters given by the original IMT-A channel model [8]. Hence, the random average powers for the $n$th cluster at time $t$ based on the time-varying delays calculated above, can be expressed as

$$
P_{n}^{\prime}(t)=e^{\frac{\left(1-r_{D S}\right)\left(\tau_{n}(t)\right)}{r_{D S} \cdot \sigma_{D S}}} \times 10^{-\frac{\xi_{n}}{10}}
$$

where $r_{D S}$ is the delay distribution proportionality factor, $\sigma_{D S}$ is delay spread, and $\xi_{n}$ is the per cluster shadowing term in $\mathrm{dB}$ and they all can be obtained from Table A1-7 in [8]. Then, 
the calculated powers are usually normalized so that the total power for all cluster powers is equal to one, i.e.,

$$
P_{n}(t)=\frac{P_{n}^{\prime}(t)}{\sum_{n=1}^{N(t)} P_{n}^{\prime}(t)}
$$

\section{E. Time-varying AoDs, $\phi_{n, m}(t)$}

In terms of the interaction of relevant parameters, referring to the derivation in the Appendix A, the time-varying function of AoD $\phi_{n, m}(t)$ can be expressed as follows.

1) if $-\pi \leq \phi_{n, m}\left(t_{0}\right)<0$,

$$
\begin{aligned}
& \phi_{n, m}(t)=\phi_{n, m}\left(t_{0}\right)-\alpha_{n, m}(t), \\
& \text { if }-\pi \leq \theta_{A} \leq \phi_{n, m}\left(t_{0}\right) \cup \phi_{n, m}\left(t_{0}\right)+\pi \leq \theta_{A}<\pi \\
& \phi_{n, m}(t)=\phi_{n, m}\left(t_{0}\right)+\alpha_{n, m}(t), \\
& \text { if } \phi_{n, m}\left(t_{0}\right)<\theta_{A}<\phi_{n, m}\left(t_{0}\right)+\pi . \\
& \text { 2) if } 0 \leq \phi_{n, m}\left(t_{0}\right)<\pi, \\
& \phi_{n, m}(t)=\phi_{n, m}\left(t_{0}\right)+\alpha_{n, m}(t), \\
& \text { if }-\pi \leq \theta_{A} \leq \phi_{n, m}\left(t_{0}\right)-\pi \cup \phi_{n, m}\left(t_{0}\right) \leq \theta_{A}<\pi \\
& \phi_{n, m}(t)=\phi_{n, m}\left(t_{0}\right)-\alpha_{n, m}(t), \\
& \text { if } \phi_{n, m}\left(t_{0}\right)-\pi<\theta_{A}<\phi_{n, m}\left(t_{0}\right)
\end{aligned}
$$

where

$$
\alpha_{n, m}(t)=
$$

$\arccos \frac{D_{\mathrm{BS}}\left(t_{0}\right)+v_{A} t \cos \left(\phi_{n, m}\left(t_{0}\right)-\theta_{A}\right)}{\sqrt{D_{\mathrm{BS}}^{2}\left(t_{0}\right)+\left(v_{A} t\right)^{2}+2 D_{\mathrm{BS}}\left(t_{0}\right) v_{A} t \cos \left(\phi_{n, m}\left(t_{0}\right)-\theta_{A}\right)}}$

\section{F. Time-varying AoAs, $\varphi_{n, m}(t)$}

Similarly, based on the interaction of relevant parameters, referring to the derivation in the Appendix $\mathrm{B}$, the time-varying function of AoA $\varphi_{n, m}(t)$ can be expressed as follows.

1) If $-\pi \leq \varphi_{n, m}\left(t_{0}\right)<0$,

$$
\begin{aligned}
& \varphi_{n, m}(t)=\theta_{v}-\gamma_{n, m}(t) \pm \pi \\
& \text { if }-\pi \leq \theta_{v}<\varphi_{n, m}\left(t_{0}\right) \cup \pi+\varphi_{n, m}\left(t_{0}\right)<\theta_{v}<\pi \\
& \varphi_{n, m}(t)=\theta_{v}+\gamma_{n, m}(t) \pm \pi \\
& \text { if } \varphi_{n, m}\left(t_{0}\right) \leq \theta_{v} \leq \pi+\varphi_{n, m}\left(t_{0}\right) \\
& \text { 2) if } 0 \leq \varphi_{n, m}\left(t_{0}\right)<\pi \\
& \varphi_{n, m}(t)=\theta_{v}+\gamma_{n, m}(t) \pm \pi \\
& \text { if }-\pi \leq \theta_{v}<\varphi_{n, m}\left(t_{0}\right)-\pi \cup \varphi_{n, m}\left(t_{0}\right)<\theta_{v}<\pi \\
& \varphi_{n, m}(t)=\theta_{v}-\gamma_{n, m}(t) \pm \pi \\
& \text { if } \varphi_{n, m}\left(t_{0}\right)-\pi \leq \theta_{v} \leq \varphi_{n, m}\left(t_{0}\right)
\end{aligned}
$$

where

$$
\begin{gathered}
v=v_{M S / Z}=\left\|\vec{v}_{M S / Z}\right\| \\
\theta_{v}=\theta_{M S / Z}=\arg \left(\vec{v}_{M S / Z}\right)
\end{gathered}
$$

$\gamma_{n, m}(t)=$

$$
\arccos \frac{v t-D_{\mathrm{MS}}\left(t_{0}\right) \cos \left(\theta_{v}-\varphi_{n, m}\right)}{\sqrt{D_{\mathrm{MS}}^{2}\left(t_{0}\right)+(v t)^{2}-2 D_{\mathrm{MS}}\left(t_{0}\right) v t \cos \left(\theta_{v}-\varphi_{n, m}\right)}} .
$$

It is worth mentioning that " $\pm \pi$ " in (27) - (30) is the corrector to ensure $\varphi_{n, m}(t)$ within the range of $[-\pi, \pi)$.

\section{STATISTICAL PROPERTIES OF NON-STATIONARY IMT-A MIMO CHANNEL MODELS}

\section{A. Derivations of local spatial CCF}

For WSSUS channels, the spatial CCF depends only on the relative BS and MS antenna element spacings, $\Delta d_{s}=$ $\left|d_{s_{1}}-d_{s_{2}}\right|$ and $\Delta d_{u}=\left|d_{u_{1}}-d_{u_{2}}\right|$, respectively. However, for non-stationary systems, the spatial CCF does not only depend on the relative antenna spacings, but also on time $t$. Due to this dependency, spatial CCF of non-stationary channel model is called local spatial CCF and can be expressed as [31]

$$
\begin{aligned}
& \rho_{s_{2} u_{2}, n}^{s_{1} u_{1}}\left(t, \triangle d_{s}, \Delta d_{u}\right)=\mathbf{E}\left\{h_{u_{1}, s_{1}, n}(t) h_{u_{2}, s_{2}, n}^{*}(t)\right\} \\
& =\frac{1}{M} \sum_{m=1}^{M} \mathbf{E}\left\{P_{n}(t) e^{j k \triangle d_{s} \sin \left(\phi_{n, m}(t)\right)} e^{j k \triangle d_{u} \sin \left(\varphi_{n, m}(t)\right)}\right\}
\end{aligned}
$$

where $(\cdot)^{*}$ denotes the complex conjugation operation and $\mathbf{E}\{\cdot\}$ designates the statistical expectation operator.

Under the WSSUS assumption, the AoAs and AoDs are considered as time-invariant. In this case, the spatial CCF will be reduced to [35]

$$
\begin{array}{r}
\rho_{s_{2} u_{2}, n}^{s_{1} u_{1}}\left(\Delta d_{s}, \Delta d_{u}\right)=\mathbf{E}\left\{h_{u_{1}, s_{1}, n}(t) h_{u_{2}, s_{2}, n}^{*}(t)\right\} \\
=\frac{P_{n}}{M} \sum_{m=1}^{M} \mathbf{E}\left\{e^{j k \triangle d_{s} \sin \left(\phi_{n, m}\right)} e^{j k \triangle d_{u} \sin \left(\varphi_{n, m}\right)}\right\} .
\end{array}
$$

\section{B. Derivations of local temporal ACF}

Considering the birth/death process, a cluster has a probability of $e^{-\lambda_{R}\left(v_{M S} \Delta t+P_{c}\left(v_{Z}+v_{A}\right) \Delta t\right)}$ to survive from $(t-\Delta t)$ to $t$. Therefore, the local temporal ACF of the non-stationary IMT-A channel model of the $n t h$ cluster can be expressed as

$r_{n}(t, \Delta t)=\mathbf{E}\left\{h_{u, s, n}(t) h_{u, s, n}^{*}(t-\Delta t)\right\}$

$=e^{-\lambda_{R}\left(v_{M S} \Delta t+P_{c}\left(v_{Z}+v_{A}\right) \Delta t\right)}$

$\times \frac{1}{M} \sum_{m=1}^{M} \mathbf{E}\left\{P_{n}(t) A_{\varphi_{n, m}}(t, \Delta t) B_{\phi_{n, m}}(t, \Delta t) C_{\varphi_{n, m}}(t, \Delta t)\right\}$

where

$$
\begin{aligned}
A_{\varphi_{n, m}}(t, \Delta t) & =e^{j k d_{u}\left[\sin \left(\varphi_{n, m}(t)\right)-\sin \left(\varphi_{n, m}(t+\Delta t)\right)\right]} \\
B_{\phi_{n, m}}(t, \Delta t)= & e^{j k d_{s}\left[\sin \left(\phi_{n, m}(t)\right)-\sin \left(\phi_{n, m}(t+\Delta t)\right)\right]} \\
C_{\varphi_{n, m}}(t, \Delta t)= & e^{-j k\|\mathrm{v}\| \cos \left(\varphi_{n, m}(t)-\theta_{v}\right)(t)} \\
& \times e^{j k\|\mathrm{v}\| \cos \left(\varphi_{n, m}(t+\Delta t)-\theta_{v}\right)(t+\Delta t)} .
\end{aligned}
$$

By applying the WSSUS assumption on (37), we get

$$
\begin{gathered}
A_{\varphi_{n, m}}(t, \Delta t)=1 \\
B_{\phi_{n, m}}(t, \Delta t)=1 \\
C_{\varphi_{n, m}}(t, \Delta t)=e^{j k\|\mathrm{v}\| \cos \left(\varphi_{n, m}-\theta_{v}\right) \Delta t} .
\end{gathered}
$$

Therefore, (36) can be written as [35]

$$
r_{n}(\Delta t)=\frac{P_{n}}{M} \sum_{m=1}^{M} \mathbf{E}\left\{e^{j k\|\mathrm{v}\| \cos \left(\varphi_{n, m}-\theta_{v}\right) \Delta t}\right\} .
$$




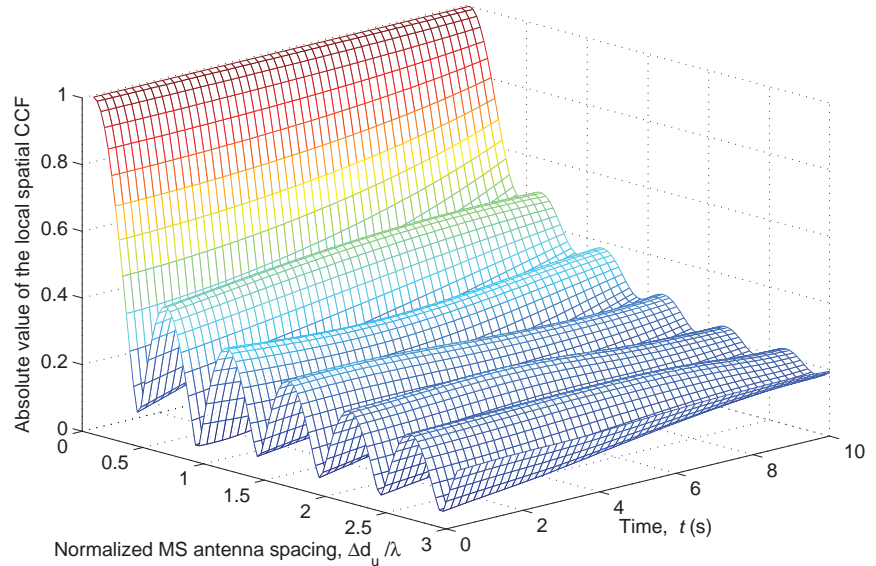

Fig. 5. The absolute value of the local spatial CCFs of the non-stationary IMTA channel model (UMa NLoS scenario, $\phi_{n, m}\left(t_{0}\right)=\operatorname{random}, D_{\mathrm{BS}}\left(t_{0}\right)=$ $100 \mathrm{~m}, \theta_{A}=15^{\circ}, v_{A}=30 \mathrm{~m} / \mathrm{s}, \varphi_{n, m}\left(t_{0}\right)=$ random, $D_{\mathrm{MS}}\left(t_{0}\right)=150 \mathrm{~m}$, $\theta_{v}=120^{\circ}$, and $v=20 \mathrm{~m} / \mathrm{s}$ ).

\section{Stationary Interval}

The stationary interval can be calculated using averaged power delay profiles (APDPs) that can be expressed as [15]

$$
\overline{P_{h}}\left(t_{k}, \tau\right)=\frac{1}{N_{P D P}} \sum_{k}^{k+N_{P D P}-1}\left|h_{u, s}\left(t_{k}, \tau\right)\right|^{2}
$$

where $N_{P D P}$ is the number of power delay profiles to be averaged, $t_{k}$ is the time of the $k$ th drop (snapshot), and $h_{u, s}\left(t_{k}, \tau\right)=\sum_{n=1}^{N} h_{u, s, n}\left(t_{k}\right) \delta\left(\tau-\tau_{n}\right)$. The correlation coefficient between two APDPs can be calculated as

$$
c\left(t_{k}, \Delta t\right)=\frac{\int \overline{P_{h}}\left(t_{k}, \tau\right) \overline{P_{h}}\left(t_{k}+\Delta t, \tau\right) \mathrm{d} \tau}{\max \left\{\int \overline{P_{h}}\left(t_{k}, \tau\right)^{2} \mathrm{~d} \tau, \int \overline{P_{h}}\left(t_{k}+\Delta t, \tau\right)^{2} \mathrm{~d} \tau\right\}} .
$$

The stationary interval can be then calculated as

$$
T_{s}\left(t_{k}\right)=\max \left\{\left.\Delta t\right|_{c\left(t_{k}, \Delta t\right) \geq c_{\text {thresh }}}\right\}
$$

where $c_{\text {thresh }}$ is a given threshold of the correlation coefficient.

\section{RESULTS AND ANALYSIS}

\section{A. Local spatial $C C F$}

By using the NLoS UMa scenario and main simulation parameters as follows: $\phi_{n, m}\left(t_{0}\right)=$ random, $D_{\mathrm{BS}}\left(t_{0}\right)=100 \mathrm{~m}$, $\theta_{A}=15^{\circ}, v_{A}=30 \mathrm{~m} / \mathrm{s}, \varphi_{n, m}\left(t_{0}\right)=$ random, $D_{\mathrm{MS}}\left(t_{0}\right)=$ $150 \mathrm{~m}, \theta_{v}=120^{\circ}$, and $v=20 \mathrm{~m} / \mathrm{s}$, Fig. 5 shows the absolute values of three-dimensional (3D) local spatial CCF of the nonstationary IMT-A channel model. It is obvious that the absolute value of the local spatial CCF changes with time $t$ due to the non-stationarity of the channel.

To highlight these changes, Fig. 6 shows two-dimensional (2D) analytical and simulated local spatial CCFs at three time instants, i.e., $t=0,5,10 \mathrm{~s}$. It is clear that simulated results match analytical ones very well, verifying the correctness of our theoretical derivations as well as simulations.

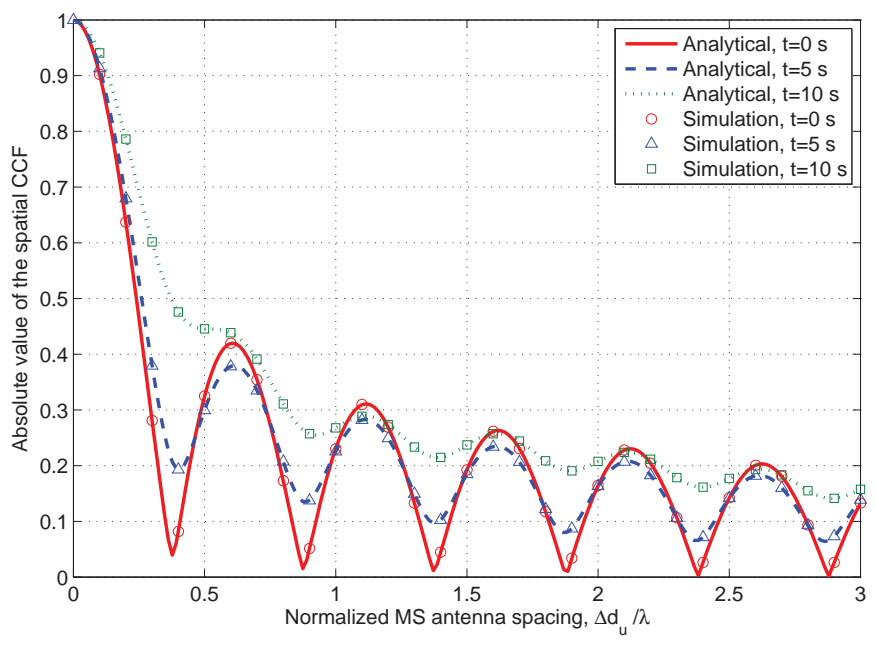

Fig. 6. The analytical and simulated local spatial CCFs of the non-stationary IMT-A at different time instants (UMa NLoS scenario, $\phi_{n, m}\left(t_{0}\right)=$ random, $D_{\mathrm{BS}}\left(t_{0}\right)=100 \mathrm{~m}, \theta_{A}=15^{\circ}, v_{A}=30 \mathrm{~m} / \mathrm{s}, \varphi_{n, m}\left(t_{0}\right)=$ random, $D_{\mathrm{MS}}\left(t_{0}\right)=150 \mathrm{~m}, \theta_{v}=120^{\circ}$, and $v=20 \mathrm{~m} / \mathrm{s}$ ).

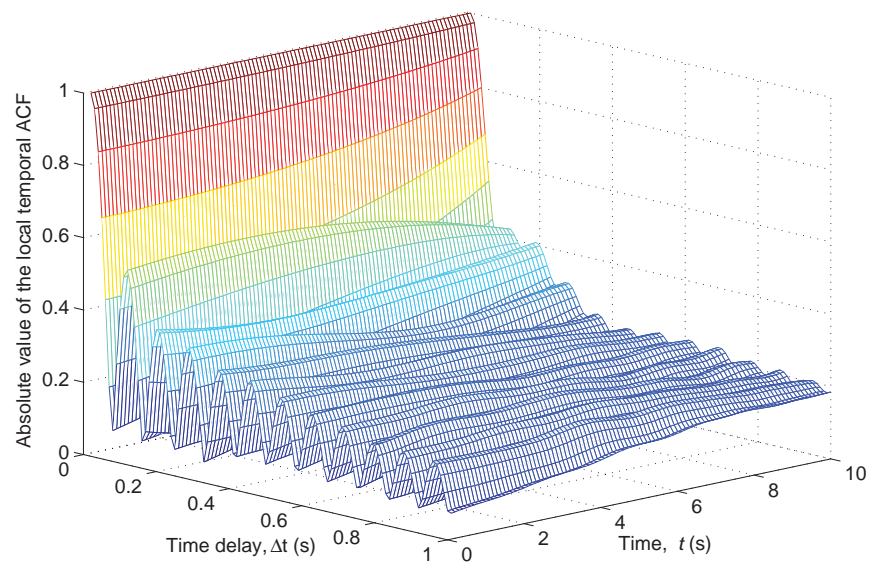

Fig. 7. The absolute value of the local temporal ACFs of the nonstationary IMT-A channel model (UMa NLoS scenario, $\phi_{n, m}\left(t_{0}\right)=$ random, $D_{\mathrm{BS}}\left(t_{0}\right)=100 \mathrm{~m}, \theta_{A}=15^{\circ}, v_{A}=30 \mathrm{~m} / \mathrm{s}, \varphi_{n, m}\left(t_{0}\right)=$ random, $D_{\mathrm{MS}}\left(t_{0}\right)=70 \mathrm{~m}, \theta_{Z}=-140^{\circ}, v_{Z}=5 \mathrm{~m} / \mathrm{s}, \theta_{M S}=120^{\circ}$, and $v_{M S}=$ $20 \mathrm{~m} / \mathrm{s})$.

\section{B. Local temporal ACF}

Still using the NLoS UMa scenario, the following parameters are used for simulating the local temporal ACF: $\phi_{n, m}\left(t_{0}\right)$ = random, $D_{\mathrm{BS}}\left(t_{0}\right)=100 \mathrm{~m}, \theta_{A}=15^{\circ}, v_{A}=30 \mathrm{~m} / \mathrm{s}$, $\varphi_{n, m}\left(t_{0}\right)=$ random, $D_{\mathrm{MS}}\left(t_{0}\right)=70 \mathrm{~m}, \theta_{Z}=-140^{\circ}, v_{Z}=5$ $\mathrm{m} / \mathrm{s}, \theta_{M S}=120^{\circ}$, and $v_{M S}=20 \mathrm{~m} / \mathrm{s}$.

Fig. 7 shows the absolute value of the 3D local temporal ACF of the non-stationary IMT-A channel model for the NLoS UMa scenario. Similar to the local spatial CCF, the absolute value of the local temporal ACF varies with time $t$ because of the time-varying AoDs and AoAs. Fig. 8 illustrates this difference by plotting the 2D analytical and simulated local temporal $\mathrm{ACF}$ at three different time instants, i.e., $t=0 \mathrm{~s}$, $t=5 \mathrm{~s}$, and $t=10 \mathrm{~s}$. Again, the simulated results match analytical results very well, verifying the correctness of our theoretical derivations as well as simulations. 


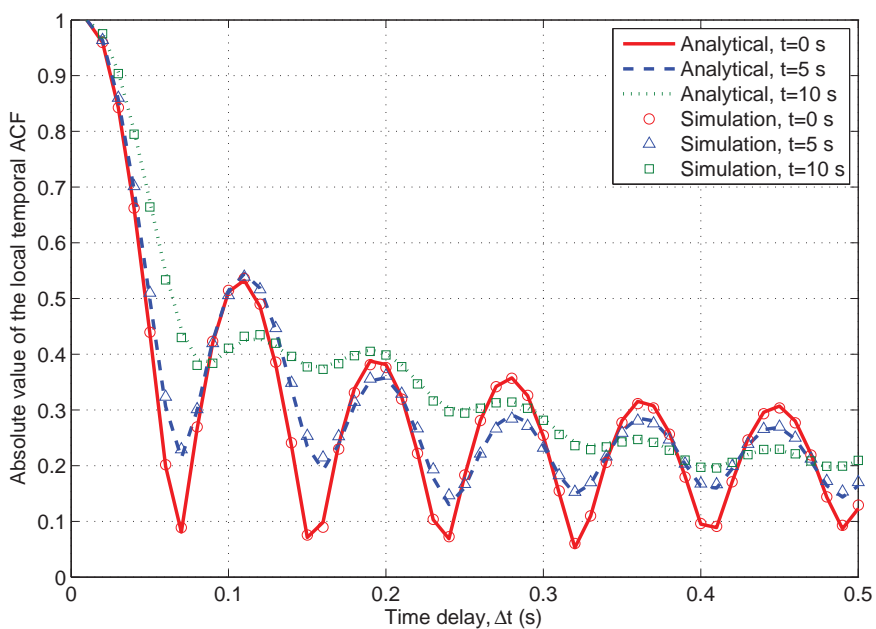

Fig. 8. The analytical and simulated local temporal ACFs of the non-stationary IMT-A at different time instants (UMa NLoS scenario, $\phi_{n, m}\left(t_{0}\right)=$ random, $D_{\mathrm{BS}}\left(t_{0}\right)=100 \mathrm{~m}, \theta_{A}=15^{\circ}, v_{A}=30 \mathrm{~m} / \mathrm{s}, \varphi_{n, m}\left(t_{0}\right)=$ random, $D_{\mathrm{MS}}\left(t_{0}\right)=70 \mathrm{~m}, \theta_{Z}=-140^{\circ}, v_{Z}=5 \mathrm{~m} / \mathrm{s}, \theta_{M S}=120^{\circ}$, and $v_{M S}=$ $20 \mathrm{~m} / \mathrm{s})$.

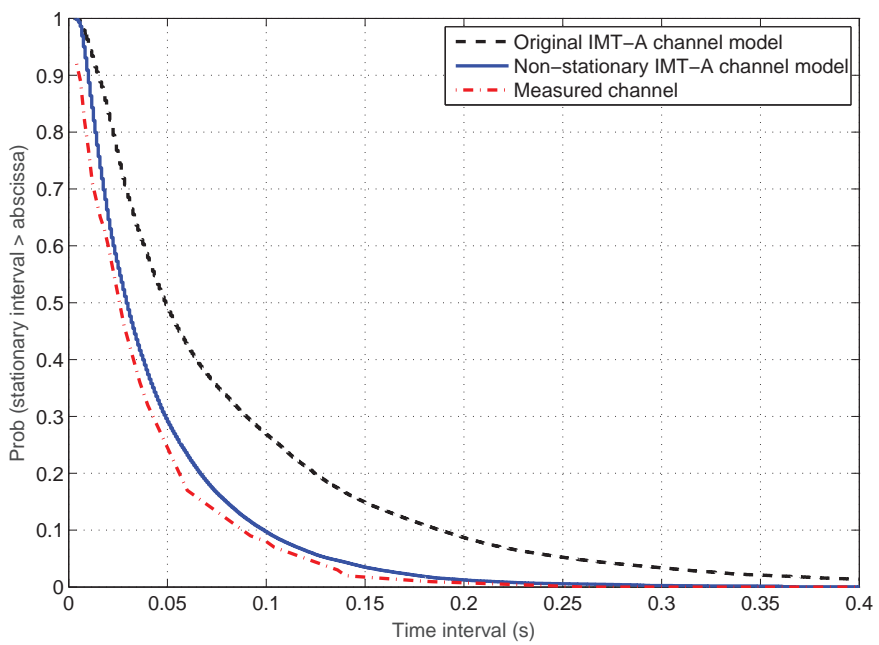

Fig. 9. The empirical CCDF of stationary intervals for the proposed nonstationary IMT-A model, the original IMT-A model, and the measured channel in [15] $\left(f_{c}=930 \mathrm{MHz}, v=90 \mathrm{~m} / \mathrm{s}, N_{P D P}=15, c_{\text {thresh }}=0.8\right)$.

\section{Stationary Interval}

Fig. 9 shows the empirical complementary cumulative distribution functions (CCDFs) of the stationary intervals for our proposed non-stationary IMT-A channel model, the original IMT-A channel model, and the measured HST channel in [15]. The simulation parameters are selected according to the measurement setup in [15] and are listed as follows: $f_{c}=$ $930 \mathrm{MHz}, v=90 \mathrm{~m} / \mathrm{s}$, and $c_{\text {thresh }}=0.8$. For the non-stationary channel model, $D_{\mathrm{BS}}\left(t_{0}\right)=100 \mathrm{~m}, v_{A}=0 \mathrm{~m} / \mathrm{s}, D_{\mathrm{MS}}\left(t_{0}\right)=$ $70 \mathrm{~m}$, and $\theta_{v}=0^{\circ}$. It is worth mentioning that since the measured HST channel is a narrowband one, in (40) we used $h_{u, s}\left(t_{k}, \tau\right)=h_{u, s, 1}\left(t_{k}\right) \delta\left(\tau-\tau_{1}\right)$ where $h_{u, s, 1}\left(t_{k}\right)$ can be obtained from (4). The good agreement between the proposed channel model and the measured channel demonstrates the feasibility of our non-stationary IMT-A channel model. From Fig. 9, the stationary interval of the proposed model is equal to

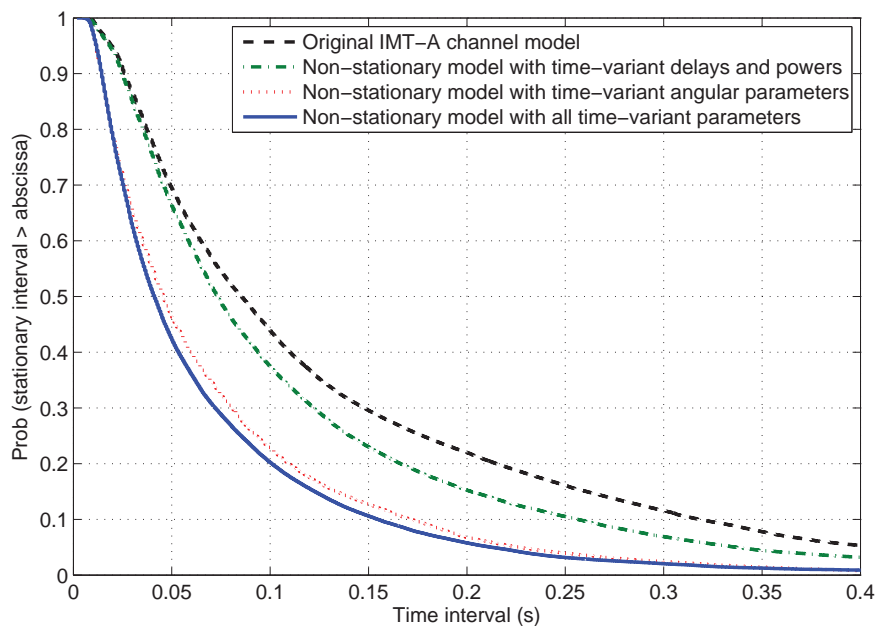

Fig. 10. The empirical CCDF of stationary intervals for the original IMTA model and the proposed non-stationary IMT-A model with all timevariant parameters, time-variant delays and powers, and time-variant angular parameters $\left(f_{c}=930 \mathrm{MHz}, v=60 \mathrm{~m} / \mathrm{s}, N_{P D P}=15, c_{\text {thresh }}=0.8\right)$.

$12.5 \mathrm{~ms}$ for $80 \%$ and $20 \mathrm{~ms}$ for $60 \%$, while the measured data is $9 \mathrm{~ms}$ for $80 \%$ and $20 \mathrm{~ms}$ for $60 \%$, which are considerably shorter than the ones of the original IMT-A channel model, i.e., $22.5 \mathrm{~ms}$ for $80 \%$ and $38.3 \mathrm{~ms}$ for $60 \%$.

To highlight the impact of the time-variant model parameters on the stationary interval, Fig. 10 shows the empirical CCDF of stationary intervals for the original IMTA channel model and the proposed non-stationary IMT-A channel model with all time-variant parameters, time-variant delays and powers, and time-variant angular parameters. The simulation parameters are the same as the ones of Fig. 9 apart from the relative speed which is $v=60 \mathrm{~m} / \mathrm{s}$. According to Fig. 9, the time-variant angular parameters have more noticeable impact on the stationary interval compared with the time-variant clusters' delays and powers.

Finally, it should also be noted that the stationary intervals of the proposed non-stationary IMT-A channel model depend on the movement parameters, e.g., the MS moving direction, the $\mathrm{MC}$ moving direction, the AoA spread, and so on.

\section{CONCLUSIONS}

This paper has proposed a non-stationary IMT-A MIMO channel model to investigate the time variation of the wireless channels in different moving scenarios by considering smallscale time-varying parameters, i.e., the number of clusters, delays, and the powers of clusters, and time-varying AoDs, AoAs. Based on the proposed non-stationary IMT-A channel model, the statistical properties in terms of the local spatial $\mathrm{CCF}$ and local temporal $\mathrm{ACF}$ have been derived. The simulation results have shown that the statistical properties would vary with time due to the time-variant parameters of the proposed channel model. The good agreement between the analytical and simulation results has verified the correctness of the analytical derivations as well as simulations. In addition, a channel characteristic called stationary interval has been investigated in terms of APDPs. Corresponding simulation 
results have proved that the stationary intervals of the proposed non-stationary IMT-A channel model match well that of the measured data and are considerably shorter than those in the original standard WSSUS IMT-A channel model, demonstrating the ability of the proposed model to accurately mimic the characteristics of high-mobility channels.

\section{APPENDIX A}

\section{DERIVATIONS OF THE TIME-VARYING AOD}

To derive the time-varying function of AoD, we assume that $\phi_{n, m}\left(t_{0}\right), D_{\mathrm{BS}}\left(t_{0}\right), \theta_{A}$, and $v_{A}$ are known, and can be obtained from a measurement campaign.

\section{A. Case A.1 $\left(-\pi \leq \phi_{n, m}\left(t_{0}\right)<0\right)$}

1) Sub-case A.1.1: $\left(-\pi \leq \theta_{A} \leq \phi_{n, m}\left(t_{0}\right) \cup \phi_{n, m}\left(t_{0}\right)+\right.$ $\left.\pi \leq \theta_{A}<\pi\right)$

In Fig. 11, the sub-case A.1.1 $\phi_{n, m}(t)$ is expressed as

$$
\phi_{n, m}(t)=\phi_{n, m}\left(t_{0}\right)-\alpha_{n, m}(t) .
$$

In trigonometry, based on the law of cosines, $\alpha_{n, m}(t)$ and $D_{\mathrm{BS}}\left(t_{0}\right)$ can be expressed as

$$
\alpha_{n, m}(t)=\arccos \frac{D_{\mathrm{BS}}^{2}\left(t_{0}\right)+D_{\mathrm{BS}}^{2}(t)-\left(v_{A} t\right)^{2}}{2 \cdot D_{\mathrm{BS}}\left(t_{0}\right) \cdot D_{\mathrm{BS}}(t)}, \alpha_{n, m}(t) \in[0, \pi]
$$

$$
D_{\mathrm{BS}}(t)=\sqrt{D_{\mathrm{BS}}^{2}\left(t_{0}\right)+\left(v_{A} t\right)^{2}-2 \cdot D_{\mathrm{BS}}\left(t_{0}\right) \cdot v_{A} t \cdot \cos \beta} .
$$

Moreover, $\beta$ can be expressed as

$$
\beta=\left(\pi+\phi_{n, m}\left(t_{0}\right)-\theta_{A}\right), \quad \beta \in[0, \pi]
$$

By substituting (45) and (46) into (44), we get

$$
\begin{aligned}
& \alpha_{n, m}(t)=\arccos \frac{D_{\mathrm{BS}}^{2}\left(t_{0}\right)+D_{\mathrm{BS}}^{2}(t)-\left(v_{A} t\right)^{2}}{2 \cdot D_{\mathrm{BS}}\left(t_{0}\right) \cdot D_{\mathrm{BS}}(t)}= \\
& \arccos \frac{D_{\mathrm{BS}}\left(t_{0}\right)+v_{A} t \cdot \cos \left(\phi_{n, m}\left(t_{0}\right)-\theta_{A}\right)}{\sqrt{D_{\mathrm{BS}}^{2}\left(t_{0}\right)+\left(v_{A} t\right)^{2}+2 \cdot D_{\mathrm{BS}}\left(t_{0}\right) \cdot v_{A} t \cdot \cos \left(\phi_{n, m}\left(t_{0}\right)-\theta_{A}\right)}}
\end{aligned}
$$

then, put (47) into (43), we finally obtain the $\phi_{n, m}(t)$.

2) Sub-case A.1.2: $\left(\phi_{n, m}\left(t_{0}\right)<\theta_{A}<\phi_{n, m}\left(t_{0}\right)+\pi\right)$

In Fig. 11, the sub-case A.1.2 $\phi_{n, m}(t)$ is expressed as

$$
\phi_{n, m}(t)=\phi_{n, m}\left(t_{0}\right)+\alpha_{n, m}(t) \text {. }
$$

Following the same procedure as in (44), (45), (46) and (47) we can express the $\phi_{n, m}(t)$ as

$$
\begin{aligned}
& \phi_{n, m}(t)=\phi_{n, m}\left(t_{0}\right)+\alpha_{n, m}(t) \\
&=\phi_{n, m}\left(t_{0}\right)+ \\
& \arccos \frac{D_{\mathrm{BS}}\left(t_{0}\right)+v_{A} t \cdot \cos \left(\phi_{n, m}\left(t_{0}\right)-\theta_{A}\right)}{\sqrt{D_{\mathrm{BS}}^{2}\left(t_{0}\right)+\left(v_{A} t\right)^{2}+2 \cdot D_{\mathrm{BS}}\left(t_{0}\right) \cdot v_{A} t \cdot \cos \left(\phi_{n, m}\left(t_{0}\right)-\theta_{A}\right)}} .
\end{aligned}
$$

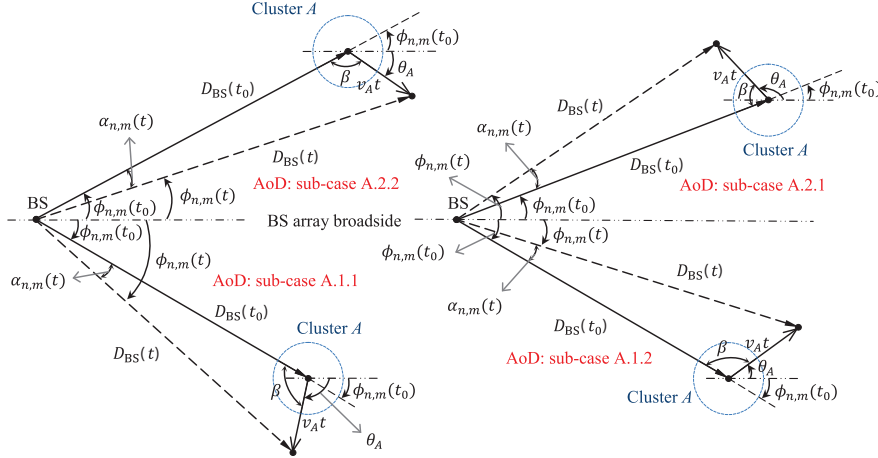

Fig. 11. Sub-case A.1.1 $\left(-\pi \leq \theta_{A} \leq \phi_{n, m}\left(t_{0}\right) \cup \phi_{n, m}\left(t_{0}\right)+\pi \leq \theta_{A}<\pi\right)$ Sub-case A.1.2 $\left(\phi_{n, m}\left(t_{0}\right)<\theta_{A}<\phi_{n, m}\left(t_{0}\right)+\pi\right)$

Sub-case A.2.1 $\left(-\pi \leq \theta_{A} \leq \phi_{n, m}\left(t_{0}\right)-\pi \cup \phi_{n, m}\left(t_{0}\right) \leq \theta_{A}<\pi\right)$

Sub-case A.2.2 $\left(\phi_{n, m}\left(t_{0}\right)-\pi<\theta_{A}<\phi_{n, m}\left(t_{0}\right)\right)$.

B. Case A.2 $\left(0 \leq \phi_{n, m}\left(t_{0}\right)<\pi\right)$

1) Sub-case A.2.1: $\left(-\pi \leq \theta_{A} \leq \phi_{n, m}\left(t_{0}\right)-\pi \cup \phi_{n, m}\left(t_{0}\right) \leq\right.$ $\left.\theta_{A}<\pi\right)$

In Fig. 11, the sub-case A.2.1 $\phi_{n, m}(t)$ is expressed as

$$
\phi_{n, m}(t)=\phi_{n, m}\left(t_{0}\right)+\alpha_{n, m}(t) .
$$

Similar to (44), (45), (46) and (47), we can write the $\phi_{n, m}(t)$ as:

$$
\begin{aligned}
& \phi_{n, m}(t)=\phi_{n, m}\left(t_{0}\right)+\alpha_{n, m}(t) \\
&=\phi_{n, m}\left(t_{0}\right)+ \\
& \arccos \frac{D_{\mathrm{BS}}\left(t_{0}\right)+v_{A} t \cdot \cos \left(\phi_{n, m}\left(t_{0}\right)-\theta_{A}\right)}{\sqrt{D_{\mathrm{BS}}^{2}\left(t_{0}\right)+\left(v_{A} t\right)^{2}+2 \cdot D_{\mathrm{BS}}\left(t_{0}\right) \cdot v_{A} t \cdot \cos \left(\phi_{n, m}\left(t_{0}\right)-\theta_{A}\right)}} .
\end{aligned}
$$

2) Sub-case A.2.2: $\left(\phi_{n, m}\left(t_{0}\right)-\pi<\theta_{A}<\phi_{n, m}\left(t_{0}\right)\right)$

In Fig. 11, the sub-case A.2.2 $\phi_{n, m}(t)$ is expressed as

$$
\phi_{n, m}(t)=\phi_{n, m}\left(t_{0}\right)-\alpha_{n, m}(t) .
$$

Following the same procedure as before, $\phi_{n, m}(t)$ can be expressed as

$$
\begin{aligned}
& \phi_{n, m}(t)=\phi_{n, m}\left(t_{0}\right)-\alpha_{n, m}(t) \\
&=\phi_{n, m}\left(t_{0}\right)- \\
& \arccos \frac{D_{\mathrm{BS}}\left(t_{0}\right)+v_{A} t \cdot \cos \left(\phi_{n, m}\left(t_{0}\right)-\theta_{A}\right)}{\sqrt{D_{\mathrm{BS}}^{2}\left(t_{0}\right)+\left(v_{A} t\right)^{2}+2 \cdot D_{\mathrm{BS}}\left(t_{0}\right) \cdot v_{A} t \cdot \cos \left(\phi_{n, m}\left(t_{0}\right)-\theta_{A}\right)}} .
\end{aligned}
$$

\section{APPENDIX B}

\section{DERIVATIONS OF THE TIME-VARYING AOA}

To derive the time-varying function of AoA with both MC $Z$ and MS are in motion, we assume that $\varphi_{n, m}\left(t_{0}\right), a\left(t_{0}\right), \theta_{v}=$ $\theta_{M S / Z}$ and $v=v_{M S / Z}$ are known.

A. Case B.1 $\left(-\pi \leq \varphi_{n, m}\left(t_{0}\right)<0\right)$

1) Sub-case B.1.1: $\left(-\pi \leq \theta_{v}<\varphi_{n, m}\left(t_{0}\right) \cup \pi+\varphi_{n, m}\left(t_{0}\right)\right.$ $\left.<\theta_{v}<\pi\right)$

In Fig. 12, the sub-case B.1.1 $\varphi_{n, m}(t)$ is expressed as

$$
\varphi_{n, m}(t)=\theta_{v}-\gamma_{n, m}(t) \pm \pi, \quad \varphi_{n, m}(t) \in[-\pi, \pi)
$$




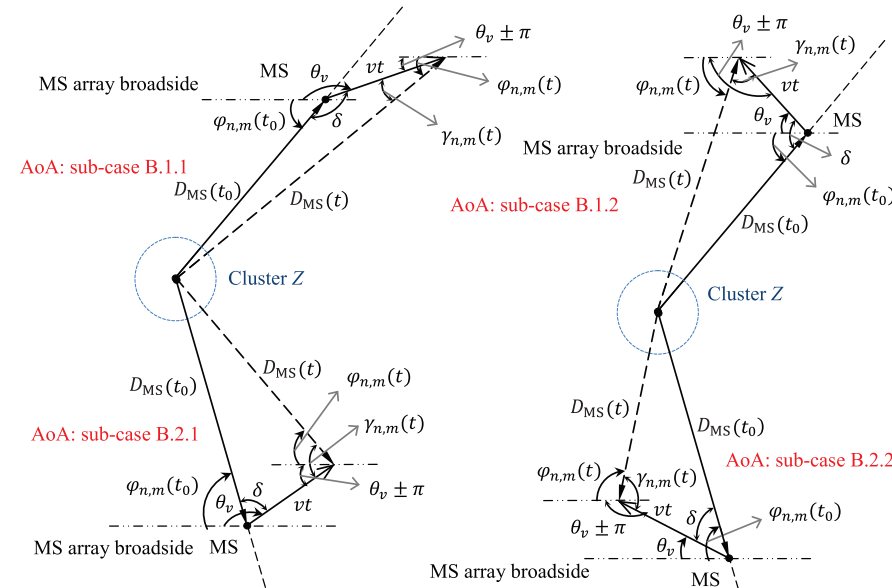

Fig. 12. Sub-case B.1.1 $\left(-\pi \leq \theta_{v}<\varphi_{n, m}\left(t_{0}\right) \cup \pi+\varphi_{n, m}\left(t_{0}\right)<\theta_{v}<\pi\right)$ Sub-case B.1.2 $\left(\varphi_{n, m}\left(t_{0}\right) \leq \theta_{v} \leq \pi+\varphi_{n, m}\left(t_{0}\right)\right)$

Sub-case B.2.1 $\left(-\pi \leq \theta_{v}<\varphi_{n, m}\left(t_{0}\right)-\pi \cup \varphi_{n, m}\left(t_{0}\right)<\theta_{v}<\pi\right)$

Sub-case B.2.2 $\left(\varphi_{n, m}\left(t_{0}\right)-\pi \leq \theta_{v} \leq \varphi_{n, m}\left(t_{0}\right)\right)$.

In trigonometry, based on the law of cosines, $\gamma_{n, m}(t)$ and $a(t)$ can be derived as:

$\gamma_{n, m}(t)=\arccos \frac{D_{\mathrm{MS}}^{2}(t)+(v t)^{2}-D_{\mathrm{MS}}^{2}\left(t_{0}\right)}{2 \cdot D_{\mathrm{MS}}(t) \cdot v t}, \gamma_{n, m}(t) \in[0, \pi]$

$D_{\mathrm{MS}}(t)=\sqrt{D_{\mathrm{MS}}^{2}\left(t_{0}\right)+(v t)^{2}-2 \cdot D_{\mathrm{MS}}\left(t_{0}\right) \cdot v t \cdot \cos \delta}$.

Moreover, $\delta$ can be expressed as:

$$
\delta=\left(\varphi_{n, m}\left(t_{0}\right)-\theta_{v}\right), \quad \delta \in[0, \pi]
$$

So, we put (56) and (57) into (55), we have

$\gamma_{n, m}(t)=\arccos \frac{D_{\mathrm{MS}}^{2}(t)+(v t)^{2}-D_{\mathrm{MS}}^{2}\left(t_{0}\right)}{2 \cdot D_{\mathrm{MS}}(t) \cdot v t}=$

$\arccos \frac{v t-D_{\mathrm{MS}}\left(t_{0}\right) \cdot \cos \left(\varphi_{n, m}\left(t_{0}\right)-\theta_{v}\right)}{\sqrt{D_{\mathrm{MS}}^{2}\left(t_{0}\right)+(v t)^{2}-2 \cdot D_{\mathrm{MS}}\left(t_{0}\right) \cdot v t \cdot \cos \left(\varphi_{n, m}\left(t_{0}\right)-\theta_{v}\right)^{[}}}$.

Therefore, putting (58) into (54), we finally obtain the $\varphi_{n, m}(t)$.

2) Sub-case B.1.2: $\left(\varphi_{n, m}\left(t_{0}\right) \leq \theta_{v} \leq \pi+\varphi_{n, m}\left(t_{0}\right)\right)$

In Fig. 12, the sub-case B.1.2 $\varphi_{n, m}(t)$ is expressed as

$$
\varphi_{n, m}(t)=\theta_{v}+\gamma_{n, m}(t) \pm \pi, \quad \varphi_{n, m}(t) \in[-\pi, \pi)
$$

and

$$
\delta=\left(\theta_{v}-\varphi_{n, m}\left(t_{0}\right)\right), \quad \delta \in[0, \pi] .
$$

By repeating the similar calculation for sub-case B1.1, we derive the $\varphi_{n, m}(t)$ where $\gamma_{n, m}(t)$ is expressed as in (58).

B. Case B.2 $\left(0 \leq \varphi_{n, m}\left(t_{0}\right)<\pi\right)$

1) Sub-case B.2.1: $\left(-\pi \leq \theta_{v}<\varphi_{n, m}\left(t_{0}\right)-\pi \cup\right.$ $\left.\varphi_{n, m}\left(t_{0}\right)<\theta_{v}<\pi\right)$

In Fig. 12, the sub-case B.2.1 $\varphi_{n, m}(t)$ is expressed as

$$
\varphi_{n, m}(t)=\theta_{v}+\gamma_{n, m}(t) \pm \pi, \quad \varphi_{n, m}(t) \in[-\pi, \pi)
$$

and

$$
\delta=\left(\theta_{v}-\varphi_{n, m}\left(t_{0}\right)\right), \quad \delta \in[0, \pi] .
$$

Again, by repeating the similar calculation for sub-case

B1.1, we derive the $\varphi_{n, m}(t)$.

In Fig. 12, the sub-case B.2.2 $\varphi_{n, m}(t)$ is expressed as

$$
\varphi_{n, m}(t)=\theta_{v}-\gamma_{n, m}(t) \pm \pi, \quad \varphi_{n, m}(t) \in[-\pi, \pi)
$$

and

$$
\delta=\left(\theta_{v}-\varphi_{n, m}\left(t_{0}\right)\right), \quad \delta \in[0, \pi] .
$$

Again, by repeating the similar calculation for sub-case B1.1, we derive the $\varphi_{n, m}(t)$.

\section{REFERENCES}

[1] V. Erceg, et al., IEEE 802.11 document 03/940r4, "TGn channel models," May 2004.

[2] 3GPP, TS 36.101, v14.1.0, "3rd generation partnership project; Technical specification group radio access network; Evolved universal terrestrial radio access (E-UTRA); User equipment (UE) radio transmission and reception (Release 14)," Sept. 2016.

[3] 3GPP TR 25.996 v13.0.0, "3rd generation partnership project; Technical specification group radio access network; Spatial channel model for multiple input multiple output (MIMO) simulations (Release 13)," Dec. 2015.

[4] D. S. Baum, J. Hansen, J. Salo, G. Del Galdo, M. Milojevi and P. Kyösti, "An interim channel model for beyond-3G systems: extending the 3GPP spatial channel model (SCM)," in Proc. IEEE VTC'05-Spring, Stockholm, Sweden, May-June 2005, pp. 3132-3136.

[5] D. S. Baum, et al., "Final report on link level and system level channel models," IST-2003-507581, WINNER D5.4 v1.4, Nov. 2005.

[6] P. Kyösti, et al., "WINNER II channel models," IST-4-027756, WINNER II D1.1.2, v1.2, Apr. 2008

[7] J. Meinila, P. Kyösti, L. Hentila, T. Jamsa, E. Suikkanen, E. Kunnari, and M. Narandzia, "D5.3: WINNER+ final channel models," CELTIC/CP5026, June 2010.

[8] ITU-R M.2135-1, "Guidelines for evaluation of radio interface technologies for IMT-Advanced," Geneva, Switzerland, Rep. ITU-R M.2135-1, Dec. 2009.

[9] K. Guan, et al., "Excess propagation loss modeling of semi-closed obstacles for intelligent transportation systems," IEEE Trans. Intell. Transp. Syst., vol. 17, no. 8, pp. 2171-2181, Aug. 2016.

[10] K. Guan, Z. Zhong, J. I. Alonso and C. Briso-Rodriguez, "Measurements of distributed antenna systems at $2.4 \mathrm{GHz}$ in a realistic subway tunnel environment," IEEE Trans. Veh. Technol., vol. 61, no. 2, pp. 834-837, Feb. 2012.

[11] O. Renaudin, V. M. Kolmonen, P. Vainikainen, and C. Oestges, "Nonstationary narrowband MIMO inter-vehicle channel characterization in the 5-GHz band," IEEE Trans. Veh. Technol., vol. 59, no. 4, pp. 2007-2015, May 2010.

[12] J. Karedal, F. Tufvesson, N. Czink, A. Paier, C. Dumard, T. Zemen, C. F. Mecklenbrauker, and A. F. Molisch, "A geometry-based stochastic MIMO model for vehicle-to-vehicle communications," IEEE Trans. Wireless Commun., vol. 8, no. 7, pp. 3646-3657, Jul. 2009.

[13] J. W. Wallace and M. A. Jensen, "Measurement and characterization of the time variation of indoor and outdoor MIMO channels at 2.4 and 5.2 GHz," in Proc. IEEE VTC'05-Fall, Dallas, USA, vol. 2, Sept. 2005, pp. 1289-1293.

[14] J. W. Wallace and M. A. Jensen, "Time-varying MIMO channels: measurement, analysis, and modeling," IEEE Trans. Antennas Propag., vol. 54, no. 11, pp. 3265-3273, Nov. 2006.

[15] B. Chen, Z. Zhong, and B. Ai, "Stationarity intervals of time-variant channel in high speed railway scenario," J. China Commun., vol. 9, no. 8, pp. 64-70, Aug. 2012.

[16] A. Paier, et al., "Non-WSSUS vehicular channel characterization in highway and urban scenarios at $5.2 \mathrm{GHz}$ using the local scattering function," in WSA'08, Darmstadt, Germany, Feb. 2008, pp. 9-15.

[17] X. Ge, J. Chen, C.-X. Wang, J. S. Thompson and J. Zhang, "5G green cellular networks considering power allocation schemes," Sci. China Inf. Sci., vol. 59, no. 2, Feb. 2016. 
[18] C.-X. Wang, X. Cheng, and D. I. Laurenson, "Vehicle-to-vehicle channel modeling and measurements: recent advances and future challenges", IEEE Commun. Mag., vol. 47, no. 11, pp. 96-103, Nov. 2009.

[19] C.-X. Wang, A. Ghazal, B. Ai, and P. Fan, "Channel measurements and models for high-speed train communication systems: a survey," IEEE Commun. Surveys Tuts, vol. 18, no. 2, pp. 974-987, 2nd Quart., 2016.

[20] C.-X. Wang, S. Wu, L. Bai, X. You, J. Wang, and C.-L. I, "Recent advances and future challenges for massive MIMO channel measurements and models," Sci. China Inf. Sci., Invited Paper, vol. 59, no. 2, pp. 1-16, Feb. 2016.

[21] L. Liu, D. W. Matolak, C. Tao, Y. Li, B. Ai, and H. Chen, "Channel capacity investigation of a linear massive MIMO system using spherical wave model in LOS scenarios," Sci. China Inf. Sci., vol. 59, no. 2, Feb. 2016.

[22] J. Karedal, F. Tufvesson, N. Czink, A. Paier, C. Dumard, T. Zemen, C. F. Mecklenbrauker, and A. F. Molisch, "Measurement-based modeling of vehicle-to-vehicle MIMO channels," in Proc. IEEE ICC'09, Dresden, Germany, June 2009, pp. 1-6.

[23] K. Guan, et al., "On the influence of scattering from traffic signs in vehicle-to-X communications," IEEE Trans. Veh. Technol., vol. 65, no. 8 , pp. 5835-5849, Aug. 2016.

[24] S. Knorzer, M. A. Baldauf, T. Fugen, and W. Wiesbeck, "Channel analysis for an OFDM-MISO train communications system using different antennas," in Proc. IEEE VTC'07-Fall, Baltimore, USA, Oct. 2007, pp. 809-813.

[25] I. Sen and D. W. Matolak, "Vehicle-vehicle channel models for the 5GHz band," IEEE Trans. Intell. Transp. Syst., vol. 9, no. 2, pp. 235-245, June 2008.

[26] S. Lin, Z. Zhong, L. Cai, and Y Luo, "Finite state Markov modelling for high speed railway wireless communication channel," in Proc. IEEE GLOBECOM'12, California, USA, Dec. 2012, pp. 5421-5426.

[27] H. Xiao, A. G. Burr, and L. Song, "A time-variant wideband spatial channel model based on the 3GPP model," in Proc. IEEE VTC'06-Fall, Montreal, Canada, Sept. 2006, pp. 1-5.

[28] A. Chelli and M. Pätzold, "A non-stationary MIMO vehicle-to-vehicle channel model based on the geometrical T-junction model," in Proc. IEEE WCSP'09, Nanjing, China, Nov. 2009, pp. 1-5.

[29] A. Chelli and M. Pätzold, "A non-stationary MIMO vehicle-to-vehicle channel model derived from the geometrical street model," in Proc. IEEE VTC'11-Fall, Budapest, Hungary, Sept. 2011, pp. 1-6.

[30] A. Ghazal, C.-X. Wang, Y. Liu, P. Fan, and M. K. Chahine, "A generic non-stationary MIMO channel model for different high-speed train scenarios," in Proc. IEEE ICCC'15, Shenzhen, China, Nov. 2015.

[31] A. Ghazal, C.-X. Wang, B. Ai, D. Yuan, and H. Haas, "A nonstationary wideband MIMO channel model for high-mobility intelligent transportation systems," IEEE Trans. Intell. Transp. Syst., vol. 16, no. 2, pp. 885-897, Apr. 2015.

[32] T. Zwick, C. Fischer, D. Didascalou, and W. Wiesbeck, "A stochastic spatial channel model based on wave-propagation modeling," IEEE J. Sel. Areas Commun., vol. 18, no. 1, pp. 6-15, Jan. 2000.

[33] S. Wu, C.-X. Wang, H. Haas, H. Aggoune, M. M. Alwakeel, and B. Ai, "A non-stationary wideband channel model for massive MIMO communication systems," IEEE Trans. Wireless Commun., vol. 14, no. 3, pp. 1434-1446, Mar. 2015.

[34] S. Wu, C.-X. Wang, H. Aggoune, M. M. Alwakeel, and Y. He, "A non-stationary 3D wideband twin-cluster model for 5G massive MIMO channels," IEEE J. Sel. Areas Commun., vol. 32, no. 6, pp. 1207-1218, June 2014.

[35] Q. Yao, Y. Yuan, A. Ghazal, C.-X. Wang, L. Luan, and X. Lu, "Comparison of the statistical properties of the LTE-A and IMT-A channel models," in Proc. IEEE WCNC'12, Paris, France, Apr. 2012.

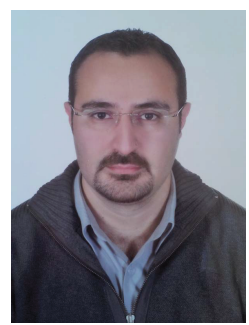

Ammar Ghazal (S'15) received the BSc degree in Electronics and Telecommunication Engineering from Damascus University, Syria in 2006, the MSc by Research from Heriot-Watt University, Edinburgh, U.K., in 2010, and the PhD degree from the University of Edinburgh and Heriot-Watt University, Edinburgh, U.K., in 2015.

$\mathrm{He}$ is now with the Centre for Electronic and Communications Engineering, De Montfort University, U.K., as an Early Career Academic Fellow. His main research interests include wireless propagation channel characterization and modeling, non-stationary channel models, high mobility wireless propagation, and advanced MIMO communication technologies.

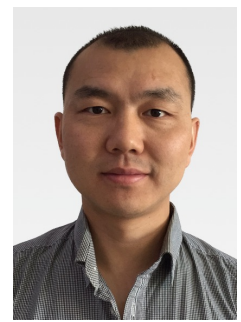

Yi Yuan received the B.Sc. degree (with distinction) in electronic engineering from Tianjin University of Technology, Tianjin, China, in 2006, the M.Sc. degree (with distinction) in mobile communications from Heriot-Watt University, Edinburgh, U.K., in 2009, and the joint Ph.D. degree from The University of Edinburgh and Heriot-Watt University, Edinburgh, U.K., in 2014.

He has been with Ocado Technology, U.K., as a Wireless Engineer-Specialist/R\&D, since 2014. His main research interests include industrial wireless control systems, wireless network optimization, remote sensor system, mobileto-mobile communications and advanced wireless MIMO channel modeling and simulation.

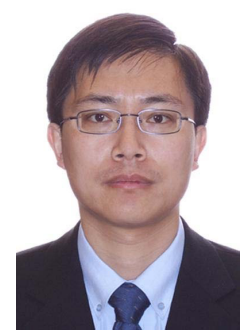

Cheng-Xiang Wang (S'01-M'05-SM'08) received the BSc and MEng degrees in Communication and Information Systems from Shandong University, China, in 1997 and 2000, respectively, and the $\mathrm{PhD}$ degree in Wireless Communications from Aalborg University, Denmark, in 2004

$\mathrm{He}$ has been with Heriot-Watt University, Edinburgh, U.K., since 2005 and was promoted to a Professor in 2011. He is also an Honorary Fellow of the University of Edinburgh, U.K., and a Chair/Guest Professor of Shandong University and Southeast University, China. He was a Research Fellow at the University of Agder, Grimstad, Norway, from 2001-2005, a Visiting Researcher at Siemens AG Mobile Phones, Munich, Germany, in 2004, and a Research Assistant at Hamburg University of Technology, Hamburg, Germany, from 2000-2001. His current research interests include wireless channel modeling and 5G wireless communication networks, including green communications, cognitive radio networks, high mobility communication networks, massive MIMO, millimeter wave communications, and visible light communications. He has published 1 book, 1 book chapter, and about 260 papers in refereed journals and conference proceedings.

Prof. Wang served or is currently serving as an editor for 12 international journals, including the IEEE TRANSACTIONS ON VEHICULAR TECHNOLOGY (since 2011), IEEE TRANSACTIONS ON COMMUNICATIONS (since 2015), and IEEE TRANSACTIONS ON WIRELESS COMMUNICATIONS (2007-2009). He was the leading Guest Editor for IEEE JOURNAL ON SELECTED AREAS IN COMMUNICATIONS, Special Issue on Vehicular Communications and Networks. He served or is serving as a TPC member, TPC Chair, and General Chair for over 80 international conferences. He received 8 Best Paper Awards from IEEE Globecom 2010, IEEE ICCT 2011, ITST 2012, IEEE VTC 2013-Spring, IWCMC 2015, IWCMC 2016, and IEEE/CIC ICCC 2016. He is a Fellow of the IET and HEA, and a member of the EPSRC Peer Review College. 


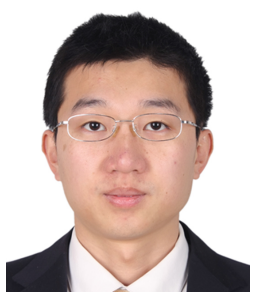

Yan Zhang (S'06-M'10) received the B.Sc. degree in information engineering from the Beijing Institute of Technology, Beijing, China, in 2005, and the Ph.D. degree in information and communication engineering from Tsinghua University, Beijing, China, in 2010 .

From July 2010 to June 2013, he was with the Department of Electronic Engineering, Tsinghua University, as a Postdoctoral Researcher. From August 2014 to August 2015, he was a Research Assistant with the School of Engineering and Physical Sciences, Heriot-Watt University, Edinburgh, U.K. He is currently an Associate Professor with the School of Information and Electronics, Beijing Institute of Technology, Beijing, China. His research interests include wireless channel modeling, physical security, and 5G mobile communication systems.

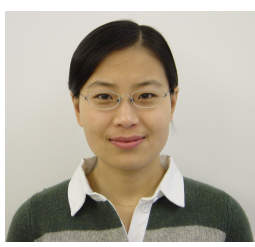

Qi Yao received the BSc and MEng degrees in Communication and Information Systems from Shandong University, China, in 1997 and 2000, respectively.

She was a Student Research Assistant with the Department of Communication Networks, Hamburg University of Technology, Hamburg, Germany from 2000-2001, a Research Fellow at the University of Agder, Grimstad, Norway, from 2001-2005, and a Research Associate at Heriot-Watt University from 2011-2012. Her research interests include wireless channel modeling, high-mobility communications, and channel parameter estimation.

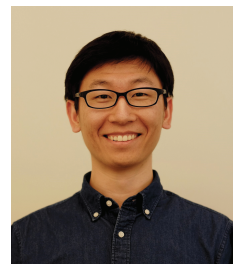

Hongrui Zhou received the B.Sc. degree in communication engineering from Xidian University, Xi'an, China, in 2005, the M.Sc. degree in communication and information systems from Xidian University, Xi' an, China, in 2008.

$\mathrm{He}$ is working at Huawei Technologies Sweden $\mathrm{AB}, \mathrm{R} \& \mathrm{D}$ Center now. His main research interests include advance radio resources management algorithm for $5 \mathrm{G}$ mobile broadband systems, Massive MIMO channel modeling and simulation.

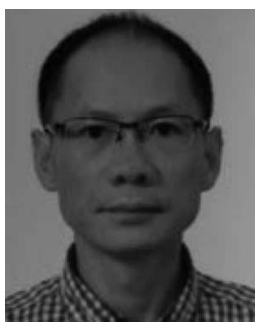

Weiming Duan received the M.Sc. degree from the University of Electronic Science and Technology of China, in 1999, and joined Huawei in the same year.

His research interests include WCDMA chipset algorithm design, WCDMA UE positioning, LTE system performance evaluation, advanced receiver research, and the 5G air-interface research. 